\title{
On a conjecture of Mahler
}

\section{V.C. Dumir and R.J. Hans-Gill}

Let $R$ be the field of real numbers. For $\alpha$ in $R$, let $\|\alpha\|$ be the distance of $a$ from the nearest integer. The following conjecture of Kurt Mahler [BuzZ. Austral. Math. Soc. 14 (1976), 463-465] is proved.

Let $m, n$ be two positive integers $n \geq 2 m$. Let $S$ be a finite or infinite set of positive integers with the following properties:

(Q1) $S$ contains the integers $m, m+1, \ldots, n-m$;

(Q2) every element of $S$ satisfies

$$
\|s / n\| \geq m / n \text {. }
$$

Then

$$
\sup _{\alpha \in R} \inf \|s \alpha\|=m / n \text {. }
$$

1. Introduction

Let $R$ be the field of real numbers. For $\alpha \in R$, let $\|\alpha\|$ be the distance of $\alpha$ from the nearest integer. Mahler [3] has proved the following:

THEOREM. Let $S$ be a finite or infinite set of positive integers with the following two properties:

(i) $S$ contains the integers $1,2, \ldots, n-1$;

(ii) $S$ does not contain any multiple of $n$.

Received 23 September 1976. Communicated by Kurt Mahler. The authors are grateful to Professor R.P. Bambah for some useful comments. 
Then

$$
\sup _{\alpha \in R} \inf _{s \in S}\|s \alpha\|=I / n \text {. }
$$

Mahler also conjectured the following generalization.

CONJECTURE. Let $m, n$ be two positive integers such that $2 m \leq n$.

Let $S$ be a finite or infinite set of positive integers with the following two properties:

$\left(Q_{1}\right) S$ contains the integers $m, m+1, \ldots, n-m$;

$\left(Q_{2}\right)$ every element of $S$ satisfies the inequality

$$
\|s / n\| \geq m / n \text {. }
$$

Then

$$
\sup _{\alpha \in R} \inf \|s \alpha\|=m / n \text {. }
$$

Our object in this note is to prove the above conjecture.

$$
2 \text {. }
$$

We shall use the following:

LEMMA. Let $m$ be a positive integer. Let $K$ be a convex body in the $n$-dimensional euclidean space $R_{n}$ with centre 0 and volume $V(K)>2^{n} m$. Then there are $m$ non-zero points $X_{1}, \ldots, X_{m}$ of the integral lattice such that

$$
\begin{aligned}
& \text { (i) } x_{i} \in K, 1 \leq i \leq m, \\
& \text { (ii) } x_{i}-x_{j} \in K, 1 \leq i, j \leq m, \\
& \text { (iii) } 0<x_{1}<x_{2}<\ldots<x_{m} \text {, where }<\text { is the lexicographic } \\
& \text { ordering in } R_{n} .
\end{aligned}
$$

REMARK. This result is essentially due to van der Corput [1], but he did not bring out the fact that the points $X_{i}$ can be chosen to satisfy (ii) and (iii) also. Here we indicate the necessary modifications to ensure (ii) and (iii). 
Proof. van der Corput's result rests on the fact that $V\left(\frac{1}{2} K\right)>m$ implies the existence of a point $Z \in R_{n}$ and $(m+1)$ distinct points $Y_{0}, Y_{1}, \ldots, Y_{m}$ such that $Z \in \frac{1}{2} K+Y_{i}, 0 \leq i \leq m$ (see, for example, Lekkerkerker [2], p. 44). We can suppose that the points $Y_{i}$ are arranged in the lexicographic order

$$
y_{0}<y_{1}<\ldots<y_{m} .
$$

Let

$$
X_{i}=Y_{i}-Y_{0}, 1 \leq i \leq m \text {. }
$$

Then $0<x_{1}<x_{2}<\ldots<x_{m}$. Also since $\frac{1}{2} K$ is symmetric convex with centre 0 , we have

$$
X_{i}=2 \frac{\left(z-Y_{0}\right)-\left(z-Y_{i}\right)}{2} \in K
$$

and

$$
X_{i}-X_{j}=Y_{i}-Y_{j}=2 \frac{\left(Z-Y_{j}\right)-\left(Z-Y_{i}\right)}{2} \in K .
$$

This proves the lemma.

3.

Proof of Conjecture. For $\alpha=1 / n$ the condition ( $\left.Q_{2}\right)$ implies that $\inf _{s \in S}\|s \alpha\| \geq m / n$.

Therefore

$$
\sup _{\alpha \in R} \inf _{s \in S}\|s \alpha\| \geq m / n \text {. }
$$

It remains to prove that for every $\alpha \in R$,

$$
\inf _{s \in S}\|s \alpha\| \leq m / n \text {. }
$$

Let $T=\{m, m+1, \ldots, n-m\}$.

Since $S \supset T$ it suffices to prove that there is a $t \in T$ such that

$$
\|t \alpha\| \leq m / n \text {. }
$$


If $n=2 m$, then $T=\{m\}$ and $\|m a\| \leq \frac{1}{2}=m / n$ for every real number $\alpha$.

Let $n>2 m$. Let $0<\varepsilon<(n / 2 m)-1$, so that $\frac{m(1+\varepsilon)}{n}<\frac{1}{2}$. Since $T$ is a finite set it is enough to prove that for every such $\varepsilon$, there is a $t \in T$ such that

$$
\|\operatorname{ta}\|<\frac{m(1+\varepsilon)}{n}
$$

Consider the parallelogram $\Pi$ with centre 0 defined by

$$
\begin{gathered}
|\alpha x-y|<\frac{m(1+\varepsilon)}{n}, \\
|x|<n .
\end{gathered}
$$

If $(x, y)$ is an integral poin' in $\Pi$, then clearly

$$
\|x \alpha\|<\frac{m(1+\varepsilon)}{n} \text {. }
$$

The area of $\pi$ is equal to $4 m(1+\varepsilon)>4 m$. By the lemma it follows that $\Pi$ contains $m$ non-zero integral points

$$
x_{i}=\left(x_{i}, y_{i}\right), i=1,2, \ldots, m, 0 \leq x_{1} \leq x_{2} \leq \ldots \leq x_{m},
$$

and

$$
x_{i}-x_{j} \in \Pi \text { for } 1 \leq i, j \leq m \text {. }
$$

We observe that

(i) $x_{i}>0$ for each $i$, because otherwise (2) implies that

$$
\left|y_{i}\right|<\frac{m(1+\varepsilon)}{n}<\frac{2}{2} \text { and hence } y_{i}=0 \text {; }
$$

(ii) $x_{i} \neq x_{j}$ when $i \neq j$, because $x_{i}=x_{j}$ implies, by (2), that $\left|y_{i}-y_{j}\right|<\frac{2 m(1+\varepsilon)}{n}<1$ and hence $y_{i}=y_{j}$; that is, $x_{i}=X_{j}$ so that $i=j$.

Hence

$$
1 \leq x_{1}<x_{2}<\ldots<x_{m} \leq n-1 \text {. }
$$


If $x_{i} \in T$ for some $i$, then $\left\|x_{i} \alpha\right\|<\frac{m(1+\varepsilon)}{n}$ and the result follows. Suppose that $x_{i} \notin T$ for $i=1,2, \ldots, m$. Let

$$
1 \leq x_{1}<x_{2}<\ldots<x_{\gamma}<m<n-m<x_{\gamma+1}<\ldots<x_{m} \leq n-1 .
$$

Clearly $1 \leq \gamma \leq m-1$, and $1 \leq i \leq \gamma$. If the integers $x_{i}+n-m$, $1 \leq i \leq \gamma$ are distinct from the integers $x_{\gamma+j}, 1 \leq j<m-\gamma$, then the interval $n-m+1 \leq x \leq n-1$ contains at least $\gamma+(m-\gamma)=m$ integers, which is impossible because the length of the interval is $m-2$.

Therefore there exist $i$ and $j, 1 \leq i \leq \gamma, 1 \leq j \leq m-\gamma$, such that $x_{i}+n-m=x_{\gamma+j}$. Then $X_{\gamma+j}-X_{i}=(n-m, y)$ is an integral point in II . Therefore

$$
\|(n-m) \alpha\|<\frac{m(1+\varepsilon)}{n}
$$

and $n-m \in T$. Thus the conjecture is proved.

\section{References}

[1] J.G. van der Corput, "Verallgemeinerung einer Mordellschen Beweismethode in der Geometrie der Zahlen", Acta Arith. 1 (1936), $62-66$.

[2] C.G. Lekkerkerker, Geometry of numbers (Biblictheca Mathematica, 8. Wolters-Noordhoff, Groningen; North-Holland, Amsterdam, London; 1969).

[3] Kurt Mahler, "A theorem on diophantine approximations", BuZZ. Austral. Math. Soc. 14 (1976), 463-465.

Centre for Advanced Study in Mathematics, Panjab University, Chandigarh, India. 